\title{
Arthroscopic-Assisted and Minimally Invasive Plate Osteosynthesis of Tibial Plateau Fractures
}

\author{
Wakil Ahmed ${ }^{\text {a, d }}$ (D, Shah M. Hafizur Rahman ${ }^{\mathrm{b}}$, Razib Ahmed ${ }^{\mathrm{a}}$, Maftun Ahmed ${ }^{\mathrm{a}}$, \\ Abu Junaid Muhammad Musaddeque Reza ${ }^{\mathrm{c}}$
}

\begin{abstract}
Background: Tibial plateau fractures are relatively rare fractures and have always been a challenge for orthopedic surgeons. These fractures may result in significant limitations postoperatively if not treated properly. The role of arthroscopic-assisted fixation of tibial plateau fractures has been advocated in many pieces of literature in the world during the last decades. Several studies have been published regarding arthroscopically assisted internal fixation of tibial plateau fractures. As it is an intra-articular lesion, surgical management is often challenging. We have very little data regarding these issues. The aim of this study was to assess the clinical and radiological outcome after arthroscopic-assisted minimally invasive plate fixation of tibial plateau fractures.
\end{abstract}

Methods: This was a descriptive observational study which was conducted in a single Specialized Hospital, Dhaka, Bangladesh during the period from January 2015 to December 2019. Totally 38 patients with tibial plateau fractures (Schatzker type I-VI) were enrolled in the study. Radiography and 3D computed tomography were used to assess the fracture patterns. Arthroscopic-assisted minimally invasive plate fixation was done in all fractures under the image intensifier. An immediate postoperative radiograph was done and then repeated at 6 weeks as well as 3, 6, and 12 months after surgery. All data were processed, analyzed, and disseminated by MS Office and SPSS version as per need. Rasmunssen clinical and radiological assessment criteria were used for evaluation.

Results: In our study, in analyzing the Rasmunssen clinical outcome we found, the highest number of participants got an "excellent" outcome, which was present in more than $70 \%(\mathrm{n}=27)$. Besides these, $21.05 \%$ ( =8) participants got "good" and the rest $7.89 \%(\mathrm{n}=3)$ participants got "fair" outcomes. On the other hand, in analyzing the Rasmunssen radiological outcome we found, the highest number of participants got an

Manuscript submitted October 22, 2021, accepted December 9, 2021

Published online December 31, 2021

${ }^{a}$ Orthopedic Surgery, National Institute of Traumatology and Orthopaedic Rehabilitation (NITOR), Dhaka, Bangladesh

bOrthopedic Surgery, Directorate General of Health Services (DGHS), Dhaka, Bangladesh

'Kushtia Medical College, Kushtia, Bangladesh

${ }^{\mathrm{d} C}$ Corresponding Author: Wakil Ahmed, Orthopedic Surgery, NITOR, Dhaka,

Bangladesh.Email: wakil_ortho@yahoo.com

doi: https://doi.org/10.14740/jcs448 "excellent" outcome which was more than $50 \%(\mathrm{n}=22)$. Besides these, $26.32 \%(n=10)$ participants got "good", $13.16 \%(n=5)$ participants got "fair" and the rest $2.63 \%(\mathrm{n}=1)$ participants got "poor" outcomes.

Conclusion: According to the findings of this study we can conclude that arthroscopic-assisted minimally invasive plate fixation of tibial plateau fractures may have a key role in the management of such fractures and is the treatment of choice for associated intra-articular pathology. Clinical and radiological evaluations of the patients were excellent in the large majority of the patients, and poor evaluation was non-existant at clinical evaluation. Radialogical evaluation was unsatisfactory for only a small number of patients.

Keywords: Arthroscopic-assisted; Minimally invasive; Tibial plateau fracture; Rasmussen assessment criteria

\section{Introduction}

Tibial plateau fractures, despite their rarity, have long been a source of consternation for orthopedic surgeons. Over the last few decades, many authors have advocated for the use of arthroscopic-assisted repair of tibial plateau fractures. Various research on arthroscopically aided internal fixation of tibial plateau fractures has been reported; however, definitive evidence on these topics is still few. As with other intra-articular fractures, the aim of therapy is anatomical reduction, stable fixation, and early mobilization. Arthroscopic joint surgery has progressed considerably over the last several decades, and arthroscopically aided internal fixation (ARIF) is currently a highly appealing choice among the surgical therapies available. It was first used in the 1980s [1] by Reiner, McGlynn, and Jennings and was proven to be effective in the treatment of Schatzker 1, 2, and 3 fractures with reduced morbidity rates when compared to open reduction and internal fixation (ORIF). In Schatzker 5 and 6 fractures, arthroscopy appears to improve reduction quality, making a complete arthrotomy unnecessary [2]. Tibial plateau fractures account for roughly $1 \%$ of all fractures in adults [3] and can be difficult to treat. They are primarily associated with high-energy trauma in the young population. In comparison to the Duparc-Ficat classification system and the AO classification system, the Schatzker classification is the most popular and extensively used one [4]. They are too complicated to be used in regular practice. On the other 


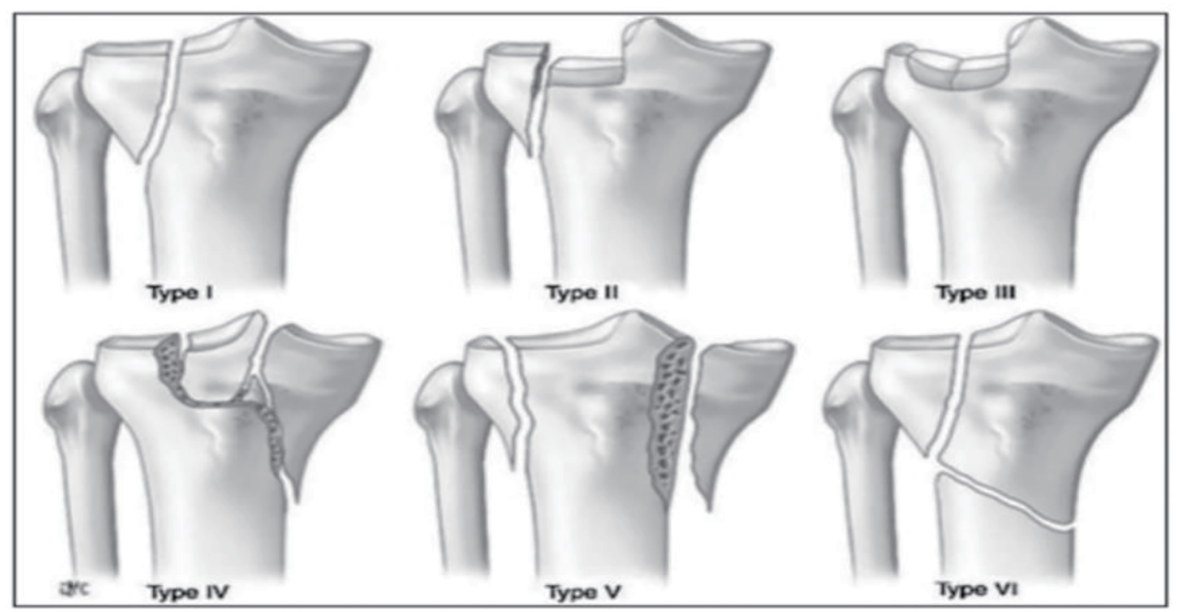

Figure 1. Schatzkar classification of tibial plateau fractures.

hand, the Schatzker classification divides the bone fractures in six simple types based on a two-dimensional representation of the fracture (Fig. 1). Each tibial plateau fracture has a unique morphology that necessitates customized treatment. It is necessary to understand the nature of trauma in order to plan treatment. These fractures are linked with a significant prevalence of soft tissue damage within the joint, demonstrating the utility of arthroscopy in their treatment. Vangsness et al observed a $47 \%$ prevalence of meniscal tears [5], Gill et al documented anterior cruciate ligament (ACL) rupture in $32 \%$ of their cases [6], and Abdel-Hamid et al discovered soft tissue damage in $71 \%$ [7]. Fractures of the intercondylar eminence account for $10 \%$ of all such fractures [2]. Approximately 5-10\% of such fractures can be connected to sports, such as skiing [6]. Cubin and Conley established categorization methods for these fractures in 1934, Marchant in 1939, Duparc and Ficat in 1960, and Hohl and Moore in 1990 [2]. Schatzker created the most frequently utilized categorization system in modern times (Fig. 1) [2]. Because of its simplicity, it was able to identify $71 \%$ of the patients who had some associated soft tissue damage. A meniscal tear was the most frequent soft tissue damage, occurring in 57\% of cases; all six forms of tibial plateau fracture had a high incidence of meniscal tears, ranging from $33 \%$ to $75 \%$, with a peripheral tear being the most common type of meniscal injury. The second most frequent injury was an ACL tear, which occurred in $25 \%$ of patients with tibial spine avulsion, accounting for $40 \%$ of ACL tears. Other ligament injuries were found in $3-5 \%$ of the cases. Type IV, V, and VI fractures had considerably greater risks of ACL and posterior cruciate ligament (PCL) damage, as well as numerous soft tissue injuries. Soft tissue injuries are prevalent in tibial plateau fractures and should be suspected in all fractures. An arthroscope can be used to diagnose these injuries [7]. Arthroscopy enables for direct observation of soft tissue and articular surface damage [8], as well as accurate reduction of fracture fragments. Arthroscopy-assisted circular external fixation of bicondylar tibial plateau fractures has just been developed [9]. This method is appropriate for Schatzker V and VI fractures because it reduces the risk of infection and soft tissue issues that might arise when two side plates are utilized [9]. Indirect reduction and external fixation have been proposed by several authors to improve the outcomes and to decrease the risk of significant complications in high-energy, complicated tibial plateau fractures [10]. The quality of reduction, ligament stability, treatment of concomitant soft-tissue injuries, and preservation of the soft-tissue envelope all contribute to successful outcomes; moreover, adequate visibility of the articular surface with minimum dissection can aid in achieving the desired aim [11]. Minimally invasive methods have gained popularity in recent years, eliminating most further surgical damage by employing a minimally invasive approach, and there are several studies with sufficient data to justify the use of arthroscopy to help in the reduction of tibial plateau fractures [11].

\section{Objectives}

The general objective was to assess the clinical and radiological outcomes among the patients with tibial plateau fractures. The specific objectives were: 1) to assess the role of arthroscopic-assisted minimally invasive fixation of tibial plateau fractures; 2) to assess the Schatzker fracture type among the patients with tibial plateau fractures; and 3) to assess the additional intra-articular lesions among the patients with tibial plateau fractures.

\section{Materials and Methods}

This was a descriptive observational study that was conducted in a single Specialized Hospital, Dhaka, Bangladesh during the period from January 2015 to December 2019. A total of 42 patients were included at the beginning, but four patients could not complete follow-up because of various reasons. Finally, 38 patients with tibial plateau fractures (Schatzker type I-VI) were enrolled in the study for analysis. Only closed fractures were included in the study. Patients who presented with associated vascular injury, compartment syndrome, and open fractures 


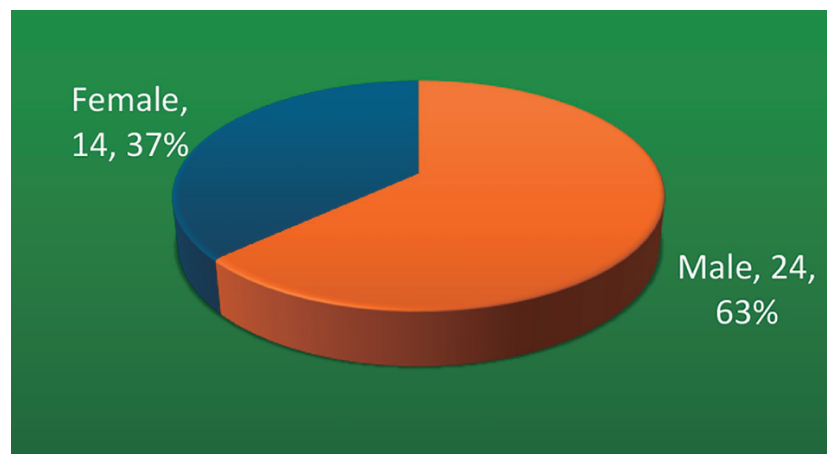

Figure 2. Gender distribution of the participants $(n=38)$.

were excluded from the study to decrease ccomplications and associated variables. Fractures were evaluated on anteroposterior (AP) and lateral radiographs and with a 3D computed tomography (CT) scan, thus the fracture pattern could be analyzed more precisely. The patients were in a supine position with the tourniquet and support at the thigh and access to the iliac crest for bone graft collection was provided when necessary. Intraoperative diagnostic arthroscopy was also performed. A standard anterolateral and anteromedial port was used for knee arthroscopy and irrigation was achieved without a pump, thus avoiding any risk of compartment syndrome. Schatzker type I fractures were reduced with pointed reduction forceps under arthroscopic visualization, type III (depression) fractures were reduced with a direct elevation of the depressed osteochondral fragment via a small cortical window, and type II (depression and separation) fractures were reduced with the elevation of the depression and fragment compression with reduction forceps. Large bone defects in the metaphysis were filled with autologous cancellous bone graft. Fractures were fixed with plates; T-plate, L-plate both locking and non-locking. Bilateral plate or dual fixation was done in more complex fractures with bicondylar involvement. Reduction and fixation were under direct visualization with arthroscopy and image intensifier. Plates were inserted sub-muscular or sub-cutaneous and extra-periosteal through a small proximal incision. The objective was the restoration of joint surface congruity to obviate the need for a large arthrotomy incision. All operation was done within 2 weeks after trauma. Among intra-articular lesions, we did the meniscal procedures (either repair or partial meniscectomy) but not the ACL or PCL reconstruction with a plan to do after the bony un-

Table 1. Schatzker Fracture Type Distribution Among Participants $(n=38)$

\begin{tabular}{lll}
\hline Schatzker type & $\mathbf{n}$ & $\mathbf{\%}$ \\
\hline I & 9 & 23.68 \\
II & 15 & 39.47 \\
III & 10 & 26.32 \\
IV & 2 & 5.26 \\
V & 1 & 2.63 \\
VI & 1 & 2.63 \\
Total & 38 & 100 \\
\hline
\end{tabular}

Table 2. Additional Intra-Articular Lesions Found Among Participants $(n=38)$

\begin{tabular}{lll}
\hline Intra-articular lesions & $\mathbf{n}$ & $\mathbf{\%}$ \\
\hline Chondral lesion & 12 & 31.58 \\
Meniscus tear & 21 & 55.26 \\
ACL rupture & 5 & 13.16 \\
Total & 38 & 100 \\
\hline
\end{tabular}

ACL: anterior cruciate ligament.

ion. An immediate postoperative radiograph was done and then repeated at 6 weeks as well as 3,6 , and 12 months after surgery. Patients were allowed a progressive partial weight-bearing with the aid of two crutches up to 6 weeks and full weight-bearing after 6 weeks. Ethical compliance was maintained with basic human-based studies, and the study was approved by the Institutional Review Board and Institutional Ethics Committee. Patients were made aware of the study, and informed consent was obtained from each participant or their legal guardians when necessary. All data were processed, analyzed, and disseminated by MS Office and SPSS version as per need.

\section{Results}

In this study, a total of 38 patients with Schatzker type I-VI tibial plateau fractures treated with arthroscopic-assisted minimally invasive fixation were evaluated. Among them, 24 were male which was $63 \%$ and 14 were female which was $37 \%$ of the total participants (Fig. 2). The mean age of the patients at the time of injury was 44 years (range $20-54$ years). In this study, according to the Schatzker fracture type analysis we observed, $23.68 \%, 39.47 \%, 26.32 \%, 5.26 \%, 2.63 \%$, and $2.63 \%$ participants were with Schatzker type I, II, III, IV, V, and VI fractures, respectively (Table 1). So, the highest number of participants were with Schatzker type II fracture which was $39.47 \%$. In analyzing the additional intra-articular lesions among the participants, we observed, $31.58 \%, 55.26 \%$ and $13.16 \%$ of participants' chondral lesions, meniscus tear and ACL rupture were associated respectively (Table 2 ). So, as an additional intra-articular lesion in the highest number of participants meniscus tear was associated which was in $55.26 \%$. In our study, in analyzing the Rasmunssen clinical outcome we found, the highest number of participants got an "excellent" outcome which was more than $71 \%(\mathrm{n}=27)$. Besides these, $21.05 \%(\mathrm{n}=8)$ participants got "good" and the rest $7.89 \%(\mathrm{n}$ =3) participants got "fair" outcomes (Table 3). In our study,

Table 3. Rasmunssen Clinical Evaluation Among Participants $(\mathrm{n}=38)$

\begin{tabular}{lll}
\hline Clinical result & $\mathbf{n}$ & $\mathbf{\%}$ \\
\hline Excellent & 27 & 71.05 \\
Good & 8 & 21.05 \\
Fair & 3 & 7.89 \\
Total & 38 & 100 \\
\hline
\end{tabular}


Table 4. Rasmunssen Radiological Evaluation Among Participants $(n=38)$

\begin{tabular}{lll} 
Radiological result & $\mathbf{n}$ & $\mathbf{\%}$ \\
\hline Excellent & 22 & 57.89 \\
Good & 10 & 26.32 \\
Fair & 5 & 13.16 \\
Poor & 1 & 2.63 \\
Total & 38 & 100 \\
\hline
\end{tabular}

in analyzing the Rasmunssen radiological outcome we found, the highest number of participants got an "excellent" outcome which was more than $57 \%(\mathrm{n}=22)$. Besides these, $26.32 \%$ $(\mathrm{n}=10)$ participants got "good", $13.16 \%(\mathrm{n}=5)$ participants got "fair" and the rest $2.63 \%(\mathrm{n}=1)$ participants got "poor" outcomes (Table 4). There were no complications directly associated with arthroscopy in any of the 38 patients.

\section{Discussion}

The aim of this study was to assess the clinical and radiological outcomes after arthroscopic-assisted minimally invasive fixation of tibial plateau fractures. Despite the various surgical methods offered by numerous authors, surgical treatment of tibial plateau fractures remains a hot topic due to complicated fracture geometry and relatively high complication rates [12]. Arthroscopy is often associated with risk factors like compartment syndrome. Compartment syndrome occurs when the pressure within a compartment rises, limiting blood supply to the region and potentially harming muscles and neurons nearby. It commonly happens in the legs, feet, arms, or hands, although it can happen anywhere in the body that has an enclosed compartment. To remove the risk of compartment syndrome in the present study, standard anterolateral and anteromedial port were used for knee arthroscopy and irrigation was achieved without a pump. The treatment objective is to get an anatomical reduction, restoration of the axis, and absolute stable internal fixation, thereby enabling an early, unrestricted passive and active range of motion. Generally, tibial plateau fractures are seen in the most productive age group due to the risk of highenergy trauma, such as road traffic accident (RTA), sports or fall from height. In the present study, the mean age of patients at the time of injury was 44 years. The minimally invasive approach is said to have a lower complication rate, a faster recovery, and a shorter hospital stay, as well as the ability to treat more intra-articular lesions [13]. In this investigation, 23 individuals had related intra-articular injuries. Hung et al argued that additional procedures such as meniscus or ligament repair during fracture reduction and fixation may be time-consuming and complex procedures with an unnecessary risk of certain complications [14]. The Canadian Orthopedic Trauma Society concluded that arthroscopically treated associated injuries may contribute to a good functional outcome [15]. We observed the concomitant treatment of meniscal injury to be reasonable and it may improve functional outcomes. In such cases, more complex procedures such as ACL reconstruction should be directed following fracture union. Because of the close closeness of fracture lines and metalwork interference, technical problems in graft attachment may arise. Another rationale of this proposal is a different rehabilitation protocol for tibial plateau fracture or ACL reconstruction [16]. In our study, according to the Schatzker fracture type analysis we observed, $23.68 \%$, $39.47 \%$ and $26.32 \%$ of participants were with Schatzker type I, II and III fractures, respectively. So, the highest number of participants were with Schatzker type II fracture which was $39.47 \%$. In our study in analyzing the additional intra-articular lesions among the participants, we observed, $31.58 \%, 55.26 \%$ and $13.16 \%$ participants chondral lesion, meniscus tear and ACL rupture were associated respectively. So, as an additional intra-articular lesion in the highest number of participants meniscus tear was associated which was in $55.26 \%$. In our study, in analyzing the Rasmunssen clinical outcome we found, the highest number of participants got an "excellent" outcome which was in more than $71 \%(\mathrm{n}=27)$. Besides these, $21.05 \%(\mathrm{n}=8)$ participants got "good" and the rest $7.89 \%(\mathrm{n}=$ 3) participants got "fair" outcomes. In our study, in analyzing the Rasmunssen radiological outcome we found, the highest number of participants got an "excellent" outcome which was in more than $57 \%(n=22)$. Besides these, $26.32 \%(n=10)$ participants got "good", $13.16 \%(\mathrm{n}=5)$ participants got "fair" and the rest $2.63 \%(n=1)$ participants got "poor" outcomes. These outcomes are comparable to those published by numerous authors [17]. In Schatzker type II and III fracture treatment, the authors found some differences in clinical results in favor of ARIF management, but these differences were not quite statistically significant [18]. According to Wang et al, both ARIF and ORIF give excellent clinical results for the treatment of Schatzker I-IV tibial plateau fractures [19]. The Rasmunssen score was used to assess clinical and radiological results in our investigation.

\section{Limitations of the study}

The study was conducted in a single hospital with a small sample size. So, the results may not represent the whole community.

\section{Conclusion and recommendations}

According to the findings of this study, we can conclude that arthroscopically assisted minimally invasive plate osteosynthesis (MIPO) of tibial plateau fracture is a safe and promising procedure and is the treatment of choice for associated intraarticular lesions. It can help to check the accuracy of reduction during fixation, diagnosis of intra-articular lesions, and their concurrent management when possible. Minimum soft tissue injury among patients going through MIPO can allow early mobilization, cut short the treatment duration, hospital stay and reduce the complications for the patient. The findings of this study may be helpful in the better treatment option of tibial plateau fractures and in similar further studies. But to get more 
reliable information we would like to recommend conducting similar more studies in several places with larger sample sizes.

\section{Acknowledgments}

None to declare.

\section{Financial Disclosure}

None to declare.

\section{Conflict of Interest}

None to declare.

\section{Informed Consent}

Informed consent was obtained from each participant or their legal guardians.

\section{Author Contributions}

Conception or design of the work: Dr. Wakil Ahmed. Data collection: Dr. Maftun Ahmed. Data analysis and interpretation: Dr. Razib Ahmed, Dr. AJM Musaddeque Reza. Drafting the article: Dr. Wakil Ahmed, Dr. Shah M. Hafizur Rahman. Critical revision of the article: Dr. Shah M. Hafizur Rahman, Dr. Razib Ahmed, Dr. AJM Musaddeque Reza. Final approval of the version to be published: Dr. Wakil Ahmed, Dr. Shah M. Hafizur Rahman.

\section{Data Availability}

Any inquiries regarding supporting data availability of this study should be directed to the corresponding author.

\section{References}

1. Jennings JE. Arthroscopic management of tibial plateau fractures. Arthroscopy. 1985;1(3):160-168.

2. Burdin G. Arthroscopic management of tibial plateau fractures: surgical technique. Orthop Traumatol Surg Res. 2013;99(1 Suppl):S208-218.

3. Buchko GM, Johnson DH. Arthroscopy assisted operative management of tibial plateau fractures. Clin Orthop Relat Res. 1996;332:29-36.

4. Muller ME, Nazarian S, Koch P. Classification AO des fractures. Berlin: Springer-Verlag. 1987:71-76.

5. Vangsness CT, Jr., Ghaderi B, Hohl M, Moore TM. Ar- throscopy of meniscal injuries with tibial plateau fractures. J Bone Joint Surg Br. 1994;76(3):488-490.

6. Gill TJ, Moezzi DM, Oates KM, Sterett WI. Arthroscopic reduction and internal fixation of tibial plateau fractures in skiing. Clin Orthop Relat Res. 20013;83:243-249.

7. Abdel-Hamid MZ, Chang CH, Chan YS, Lo YP, Huang JW, Hsu KY, Wang CJ. Arthroscopic evaluation of soft tissue injuries in tibial plateau fractures: retrospective analysis of 98 cases. Arthroscopy. 2006;22(6):669-675.

8. Wiss DA, Watson JT. Fractures of the tibial plateau. In: Rockwood CA, Green DP, Bucholz RW, Heckman JD, eds. Rockwood and Green's fractures in adults. Philadelphia:Lippincott-Raven. 1996:1920-1953.

9. Oguz E, Yanmis I, Kurklu M, Atesalp AS, Yildiz C. [The results of arthroscopically assisted circular external fixation in bicondylar tibial plateau fractures]. Acta Orthop Traumatol Turc. 2007;41(1):1-6.

10. Kataria H, Sharma N, Kanojia RK. Small wire external fixation for high-energy tibial plateau fractures. J Orthop Surg (Hong Kong). 2007;15(2):137-143.

11. Scheerlinck T, Ng CS, Handelberg F, Casteleyn PP. Medium-term results of percutaneous, arthroscopically-assisted osteosynthesis of fractures of the tibial plateau. J Bone Joint Surg Br. 1998;80(6):959-964.

12. Momaya AM, Hlavacek J, Etier B, Johannesmeyer D, Oladeji LO, Niemeier TE, Herrera N, et al. Risk factors for infection after operative fixation of Tibial plateau fractures. Injury. 2016;47(7):1501-1505.

13. Kumar A, Whittle AP. Treatment of complex (Schatzker Type VI) fractures of the tibial plateau with circular wire external fixation: retrospective case review. J Orthop Trauma. 2000;14(5):339-344.

14. Hung SS, Chao EK, Chan YS, Yuan LJ, Chung PC, Chen CY, Lee MS, et al. Arthroscopically assisted osteosynthesis for tibial plateau fractures. J Trauma. 2003;54(2):356363.

15. Zamora R, Wright C, Short A, Seligson D. Comparison between suprapatellar and parapatellar approaches for intramedullary nailing of the tibia. Cadaveric study. Injury. 2016;47(10):2087-2090.

16. Canadian Orthopaedic Trauma S. Open reduction and internal fixation compared with circular fixator application for bicondylar tibial plateau fractures. Results of a multicenter, prospective, randomized clinical trial. J Bone Joint Surg Am. 2006;88(12):2613-2623.

17. Rossi R, Bonasia DE, Blonna D, Assom M, Castoldi F. Prospective follow-up of a simple arthroscopic-assisted technique for lateral tibial plateau fractures: results at 5 years. Knee. 2008;15(5):378-383.

18. Dall'oca C, Maluta T, Lavini F, Bondi M, Micheloni GM, Bartolozzi P. Tibial plateau fractures: compared outcomes between ARIF and ORIF. Strategies Trauma Limb Reconstr. 2012;7(3):163-175.

19. Wang Z, Tang Z, Liu C, Liu J, Xu Y. Comparison of outcome of ARIF and ORIF in the treatment of tibial plateau fractures. Knee Surg Sports Traumatol Arthrosc. 2017;25(2):578-583. 